\title{
Is There More Than One Way to Talk About Sex? A Longitudinal Growth Mixture Model of Parent-Adolescent Sex Communication
}

Laura M. Padilla-Walker

Brigham Young University, laura_walker@byu.edu

Adam A. Rogers

Ryan D. McLean

Follow this and additional works at: https://scholarsarchive.byu.edu/facpub

Part of the Social and Behavioral Sciences Commons

\section{Original Publication Citation}

Padilla-Walker, L. M., Rogers, A., \& *McLean, R. (2020). Is there more than one way to talk about sex? A longitudinal growth mixture model of parent-adolescent sex communication. Journal of Adolescent Health, 67, 851-858

\section{BYU ScholarsArchive Citation}

Padilla-Walker, Laura M.; Rogers, Adam A.; and McLean, Ryan D., "Is There More Than One Way to Talk About Sex? A Longitudinal Growth Mixture Model of Parent-Adolescent Sex Communication" (2019). Faculty Publications. 4974.

https://scholarsarchive.byu.edu/facpub/4974

This Peer-Reviewed Article is brought to you for free and open access by BYU ScholarsArchive. It has been accepted for inclusion in Faculty Publications by an authorized administrator of BYU ScholarsArchive. For more information, please contact ellen_amatangelo@byu.edu. 
Original article

\title{
Is There More Than One Way to Talk About Sex? A Longitudinal Growth Mixture Model of Parent-Adolescent Sex Communication
}

\author{
Laura M. Padilla-Walker, Ph.D. *, Adam A. Rogers, Ph.D., and Ryan D. McLean \\ School of Family Life, Brigham Young University, Provo, Utah
}

Article history: Received December 11, 2019; Accepted April 22, 2020

Key words: Parent-child sex communication; Sexual risk; Longitudinal mixture model; Adolescence

\section{A B S T R A C T}

Purpose: Parents can be effective and consistent sex educators of their children, but research suggests that most parents only engage in a one-time talk about sex with their children. That being said, we know little about the potential variability in trajectories of parent-child sex communication over time. Thus, the present study took a person-centered approach to parent-child sex communication about sexual risk and explored predictors and outcomes of varying trajectories. Methods: Participants included 468 adolescents and their parents who took part in a longitudinal study every year from ages $14-18$ years (52\% female, 67\% white, and 33\% single parents).

Results: Growth mixture modeling suggested four different trajectories of parent-child sex communication using child reports and two different trajectories using mother and father reports, with the majority of parents displaying low and stable levels of communication over time. Predictors and outcomes suggested that varying trajectories were in part a function of child behaviors (i.e., early sexual debut and externalizing behaviors), and mothers who reported trajectories of moderate-stable levels of communication had children who reported safer sex practices at age 21 years.

Conclusions: The discussion focused on the benefits of longitudinal, person-centered approaches at identifying variability in parenting and the implications of findings for those concerned about the need for parental education on the importance of sex communication across adolescence.

(c) 2020 Society for Adolescent Health and Medicine. All rights reserved.

\section{IMPLICATIONS AND CONTRIBUTION}

This study provides evidence of varying trajectories of parent-child communication about sexual risk from age 14 to 18 years. A sizable minority of parents had different patterns of communication over time, suggesting the need for nuanced approaches to parent education. The findings also suggest that parents should talk to their adolescents about sex even when they are not at high risk and mothers should use less control so both parents can be involved in sex communication.
Parents are better positioned than most other adults to share sexual information with their children early in life and in developmentally appropriate ways [1], integrate sexuality conversations into normal life circumstances [2], and address sexuality and romance regularly with their children. Currently, the literature is lacking developmental perspectives that consider how parent-child sex communication changes as children move into and across adolescence, as well as the

\footnotetext{
Conflicts of interest: The authors have no conflicts of interest to disclose.

* Address correspondence to: Laura M. Padilla-Walker, Ph.D., School of Family Life, Brigham Young University, Provo, UT 84602.

E-mail address: laura_walker@byu.edu (L.M. Padilla-Walker).
}

diversity in what these trajectories might look like. Thus, in this study, we sought to explore distinct developmental trajectories in the frequency of parent-child sex communication regarding safe sex practices from ages 14 to 18 years, factors that predict this variability, and the safe sex outcomes of occupying these trajectories.

\section{Development and diversity in parent-child sex communication across adolescence}

Biological and physiological transitions during adolescence usher in a heightened interest in romance and sexuality [3]. 
These changes can create new opportunities and/or challenges for families in discussing sexuality in the home, and parents may perceive increasing demands to broach topics of sexuality in more depth and frequency. However, very little evidence is available to inform how these changing child and family dynamics may shape trajectories of parent-child sex communication across adolescence. Padilla-Walker conducted the only such study to date [4], examining the change in the frequency with which families communicated about safe sex practices from early to late adolescence, and the findings revealed very low and stable levels of sex communication across time. These findings appear to indicate that the average adolescent will receive little, if any, direct sex communication about sexual risk from his/her parents during the second decade of life.

Although informative, current patterns are limited to a description of sex communication within the average family. Parenting theories suggest that variability between families is best understood as reflecting the existence of heterogeneous typologies of parenting [5,6], and person-centered approaches have consistently verified distinct classes of parents along a number of dimensions. Therefore, it is reasonable to assume that development in various aspects of the parent-child relationship, including in sex communication, progresses with similar heterogeneity during adolescence. That is, although families may display infrequent sex communication across adolescence on average [4], there is likely to be meaningful heterogeneity in these trajectories over time. The ability to identify and predict distinct trajectories of sex communication can help scholars and practitioners locate families most in need of intervention and/or prevention efforts (i.e., families who may particularly struggle with effective sex communication).

Families occupying distinct sex communication trajectories during adolescence may be identifiable on the basis of certain parent and child factors. Literature suggests that parents who use more proactive parenting strategies that scaffold autonomy while minimizing control engage in sexual conversations with their children more regularly [7] and are more comfortable with sexual dialog [8]. Children's own behaviors also can evoke parental responses (e.g., increased solicitation/monitoring), and research has long suggested that adolescents who engage more frequently in problem and/or risky behaviors receive more frequent and punitive communication from parents [9]. Crosssectional studies have linked such parent and child inputs to sexual communication dynamics, but the degree to which these exert sustained effects on sex communication trajectories across adolescence, and meaningful variability therein, is currently unknown.

\section{Present study}

Given the lack of developmental focus in the current research on parent-child sex communication, this study had two overarching goals. First, we examined the trajectories of parent-child sex communication from ages 14 to 18 years, a period when sexual development is salient among most adolescents. We estimated these trajectories separately for adolescent, mother, and father informants, given family members' often divergent perceptions of sex communication [10,11]. Our specific hypotheses were exploratory, although we did generally predict that heterogeneity in the development of sex communication would manifest as distinct patterns of change (i.e., trajectories).
The second goal of this study was to understand the correlates of these different trajectories, both predictors and outcomes. Accumulating evidence suggests that the developmental heterogeneity in parent-child sex communication may be partly dependent on parenting strategies more broadly, as well as a child's relative level of risky behavior and/or sexual experience $[12,13]$. Therefore, we examined whether parents' autonomy support and psychological control, as well as children's externalizing problems, could predict whether families would follow distinct trajectories of sexual communication from age 14 to 18 years. We also examined the child's sexual experience, indexed by whether or not they reported having had sexual intercourse by age 14 years (start of the study), as a predictor. Finally, we examined basic demographic characteristics as predictors of occupying distinct sex communication trajectories (adolescent's biological sex, ethnic minority status, and a singleparent family structure).

In terms of outcomes, evidence from a review of primarily cross-sectional studies indicates that when parents discuss the dangers of unsafe sex (e.g., unwanted pregnancy and sexually transmitted infections), their children report less permissive sexual attitudes and intentions [14] and engage more frequently in safe sex practices (e.g., condom use) [15] and conversations with their sexual partners [16]. Therefore, it seems reasonable that trajectories of parent-child sex communication across development may aggregately predict future safe sex behaviors. Thus, we predicted that trajectories characterized by more frequent sexual communication across ages 14 to 18 years would predict safer sex behaviors in young adulthood, at age 21 years.

\section{Methods}

\section{Participants and procedures}

Participants were 468 adolescents (52\% female, 66\% white, $11 \%$ black, and 23\% multiethnic) and their mothers and 311 fathers who were part of a longitudinal study of adolescent development within the family $(\mathrm{N}=500)$. Sixty-seven percent of families were two-parent (90\% biological, 6\% stepfamilies, and $4 \%$ adoptive), and $33 \%$ were single mothers (85\% biological and $15 \%$ adopted). Forty-one percent of the sample reported $<\$ 40,000$ combined household income annually, with the average household income approximately $\$ 60,000$ annually.

Institutional review board approval was granted by Brigham Young University (Flourishing Families Project, approval number F060311). At Wave 1, participants whose children were between the ages of 11-14 years were identified using a national telephone directory and were randomly selected from a large northwestern city to reflect the demographics of local census in regards to income and ethnicity (61\% response rate, 500 families, and 468 of which were still in the study at age 14 years). Families were asked to participate in a longitudinal study of family life that consisted of a 3-hour in-home visit at each wave of data collection from Waves 1 to 5 and online questionnaires from Waves 5 to 10 . Each participant was given a $\$ 100$ visa gift card for their participation each year of in-home data collection and a $\$ 50$ visa gift card for each year of online participation. Longitudinal retention was over $90 \%$, and there were no differences in study variables between those who participated and those who dropped out of the study. Missing data were dealt with using FIML in Mplus [17], so the full sample of 468 adolescents was used in the growth mixture modeling (GMM) analysis. 


\section{Measures}

Parent-child communication about sexuality. Adolescents, mothers, and fathers responded to a 4-item measure every year from ages 14-18 years assessing parent-child communication about avoiding sexual risk (e.g., "In the last year, how often have you talked with your child/parents about preventing pregnancy?," additional items used the same stem and asked about preventing sexually transmitted diseases, using condoms, and protecting against HIV/AIDS) [15]. Items were on a scale ranging from 1 (never) to 4 (often), and higher scores indicated higher levels of parent-child communication ( $\alpha$ ranged from .91 to .95 ).

Sexual and safe sex behavior. Adolescents reported on their own sexual behaviors at the final time point (average age of 21 years) with items adapted from the Sexual Risk Survey [18]. Adolescents responded to four questions about safe sex (e.g., "the last time you had sexual intercourse, did you or your partner use a condom," addition items asked about the birth control pill, intrauterine device, or other form of protection). Affirmative responses were summed to create a safe sex score, with higher scores indicating the use of multiple forms of protection when having sexual intercourse (ranging from 0 to 4). Adolescents also reported retrospectively on the age at which they first had sexual intercourse.

Parental autonomy granting and psychological control. Adolescents responded to items about their parents' autonomy granting (five items, $\alpha=.82$; Parenting Styles and Dimensions Questionnaire) [19] and psychological control (eight items; $\alpha=.86$ ) [20] on a 1 (never) to 5 (always) scale. Sample items include "My parent encourages me to freely express myself even when I disagree with them" and "My parent tries to change how I feel or think about things."

Adolescent externalizing behavior. Adolescents responded to nine items about their own externalizing behavior on a scale ranging from 0 (not true) to 2 (often true; $\alpha=.77$ ) [21]. A sample item is "I use alcohol or drugs."

Categorical predictors. Adolescents responded to a number of items that were used as age 14 years predictors of class membership, including biological sex $(0=$ female and $1=$ male), their parents' marital status $(0=$ married and $1=$ single $)$, ethnicity ( $0=$ black or multiethnic and $1=$ white), and whether or not they had sexual intercourse by age 14 years $(0=$ no and $1=$ yes $)$.

\section{Results}

Descriptive statistics and correlations for all continuous study variables are included in Supplementary Materials. Only 8\% of the sample reported having had sexual intercourse before age 14 years, whereas at age 21 years, $73 \%$ of the sample reported having had sexual intercourse.

\section{Growth mixture model of parent-child sex communication}

Single growth curves of child-, mother- and father-reported sex communication all indicated significant variance around the intercept, slope, or quadratic term, so we moved forward with GMM [4]. We conducted three separate GMMs exploring child-, mother-, and father-reported parent-child sex communication from age 14 to 18 years.
For each model, to determine the number of classes, we examined the Bayesian Information Criterion (BIC) and the sample size-adjusted BIC (SABIC). The BIC favors models with few parameters and smaller sample sizes [22], and the SABIC adds an adjustment to reduce the sample size penalty. We also used the Lo-Mendell-Rubin adjusted likelihood ratio test, which compares two models and provides a $p$ value for $\mathrm{k}-1$ classes. Finally, we examined class sizes and took entropy (a measure of how well cases are classified) into account. Lower values indicate better fit when using BIC and SABIC, and entropy with values approaching 1 indicates clearer delineation of classes [23]. The final profile solution was selected based on goodness of fit and parsimony.

For the child-reported model, there was a clear improvement in model fit when moving from Class 1 to 2 and from Class 3 to 4 (Table 1). After considering class size, entropy, and interpretability, we selected the four-class solution. Figure 1 displays the growth trajectories of the classes, and growth parameters are in Supplementary Materials. Class $1(8 \%, \mathrm{n}=36)$ had a moderate intercept that decreased over time and then increased quite dramatically from age 16 to 18 years. We called this group of parents "Sweet 16," as it seemed they perhaps had an initial discussion about sexuality and then did not begin talking about it again until around age 16 years. Class $2(7 \%, n=32)$ had a low intercept that increased dramatically at age 16 years and then decreased dramatically by age 18 years. We called this group of parents "Peak at 16." Class $3(69 \%, \mathrm{n}=325)$ had low and stable levels of communication from age 14 to 18 years, so we called this group of parents "Low Stable Communicators." Class 4 (16\%, $\mathrm{n}=75$ ) had the highest initial level of communication, and it stayed moderate and stable over time, so we called this group of parents "Moderate Stable Communicators."

For both the mother- and father-reported models, there was a clear improvement in model fit when moving from Class 1 to 2 , but improvement was not clear when moving to any other number of classes (Table 1). Thus, after considering class size, entropy, and interpretability, we selected the two-class solution for both mothers and fathers. Figures 2 and 3 display the growth trajectories of the classes, and growth parameters are in Supplementary Materials. For both the mother- and fatherreported model, Class 1 ( $51 \%, \mathrm{n}=232$ for mothers; and $71 \%$, $\mathrm{n}=228$ for fathers) had a low initial level of communication with a gradual increase until age 16 years and then a leveling off until age 18 years. We called these parents "Low Communicators." For mothers, Class $2(49 \%, n=225)$ had moderate initial levels of communication that stayed stable across time, so we called this group "Moderate Stable Communicators." For fathers, Class $2(29 \%, n=92)$ had moderate initial levels of communication with a slight decrease until age 16 years followed by an increase back to initial levels by age 18 years. We called this group of fathers "Moderate Communicators," as the level of communication was similar to the moderate groups in the child and mother models.

\section{Predictors and outcomes of trajectories of parent-child sex communication}

Once the GMM models were established, the r3step approach in Mplus was used with class membership regressed on the predictors (marital status, child ethnicity, parental autonomy granting, parental control, child sex, child sexual activity, and child externalizing behavior), all of which were measured at age 
Table 1

Relative model fit by number of latent classes

\begin{tabular}{|c|c|c|c|c|c|}
\hline Classes & Class size $(n)$ & Entropy & BIC & SABIC & LMR \\
\hline \multicolumn{6}{|c|}{ Child report } \\
\hline 1 & 468 & - & 3912.05 & 3867.61 & - \\
\hline 2 & 108,360 & .83 & 3814.81 & 3757.68 & $p<.001$ \\
\hline 3 & $66,39,363$ & .83 & 3805.41 & 3735.59 & $p=.58$ \\
\hline 4 & $36,32,325,75$ & .85 & 3726.78 & 3644.26 & $\boldsymbol{p}<.01$ \\
\hline 5 & $34,273,87,46,28$ & .84 & 3585.83 & 3710.28 & $p=.46$ \\
\hline \multicolumn{6}{|c|}{ Mother report } \\
\hline 1 & 457 & - & 3514.64 & 3470.20 & - \\
\hline 2 & 232,225 & .72 & 3418.25 & 3354.77 & $\boldsymbol{p}<.001$ \\
\hline 3 & $164,237,56$ & .66 & 3420.50 & 3337.98 & $p=.16$ \\
\hline 4 & $65,227,159,6$ & .73 & 3424.62 & 3323.06 & $p=.27$ \\
\hline 5 & $135,55,49,5,213$ & .72 & 3445.64 & 3325.04 & $p=.48$ \\
\hline \multicolumn{6}{|c|}{ Father report } \\
\hline 1 & 320 & - & 2153.42 & 2109.01 & - \\
\hline 2 & 228,92 & .84 & 2088.31 & 2031.22 & $p<.001$ \\
\hline 3 & $50,74,196$ & .77 & 1968.41 & 1901.80 & $p=.14$ \\
\hline 4 & $86,11,162,61$ & .82 & 1900.29 & 1820.99 & $p=.30$ \\
\hline 5 & $74,159,33,15,39$ & .83 & 1894.62 & 1802.63 & $p=.06$ \\
\hline
\end{tabular}

Bolded rows represent final solutions.

$\mathrm{BIC}=$ Bayesian Information Criterion; LMR = Lo-Mendell-Rubin Adjusted Likelihood Ratio Test; SABIC = Sample Size-Adjusted Bayesian Information Criterion

14 years. Male, single, white, and had sex by age 14 years were the higher coded values. The base category was switched across regressions so that all pairwise comparisons were made. In the child-reported model, Peak at 16 (odds ratio $[\mathrm{OR}]=.93 ; p<.05$ ) and Moderate Stable $(\mathrm{OR}=1.04 ; p<.01)$ were more likely to be from single-parent homes than Low Stable Communicators, and Moderate Stable were more likely to have a child who had sex by age 14 years $(\mathrm{OR}=1.36 ; p<.01)$ than Low Stable Communicators. Peak at 16 also had lower levels of parental autonomy granting (OR $=-.87, p<.01)$, and Moderate Stable had higher levels of adolescent externalizing behavior $(\mathrm{OR}=1.50 ; p<.01)$ than did Low Stable Communicators. In the mother-reported model, Moderate Stable mothers were less likely to be white $(\mathrm{OR}=-.93 ; p<.001)$ and more likely to be single parents $(\mathrm{OR}=1.63 ; p<.001)$, have an adolescent who had sex by age 14 years $(\mathrm{OR}=1.24 ; p<.05)$, and had higher externalizing behaviors (OR $=2.01 ; p<.001$ ) compared with Low Stable Communicators. In the father-reported model, Low

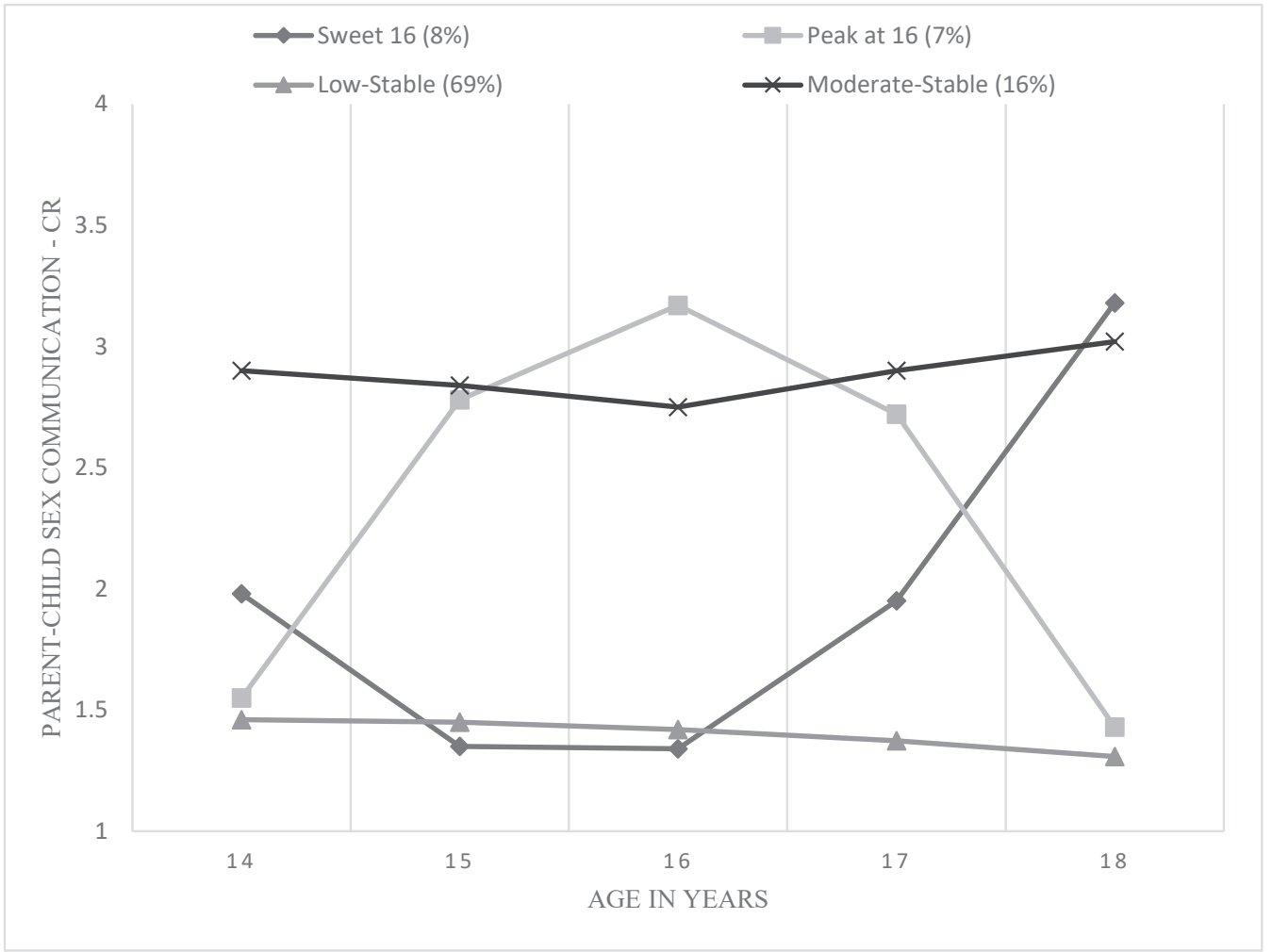

Figure 1. Growth mixture model of child-reported parent-child sex communication. 


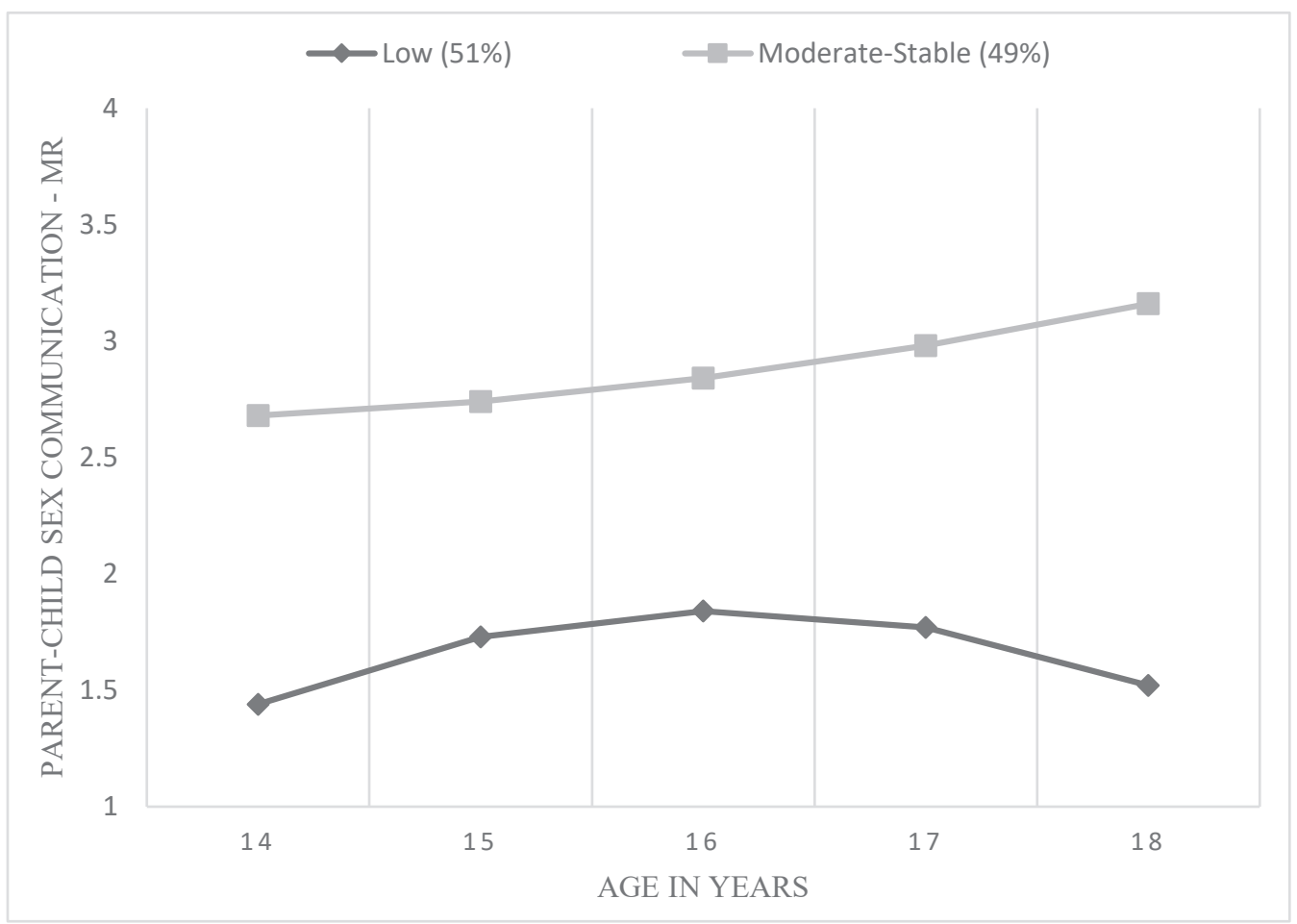

Figure 2. Growth mixture model of mother-reported parent-child sex communication.

Communicators had higher levels of maternal control $(\mathrm{OR}=.51$; $p<.001)$ but lower levels of paternal control (OR $=-.86 ; p<.01)$ compared with Moderate Communicators. Moderate communicating fathers also had adolescents with higher levels of externalizing behaviors $(\mathrm{OR}=1.91 ; p<.001)$ compared with Low Communicators.

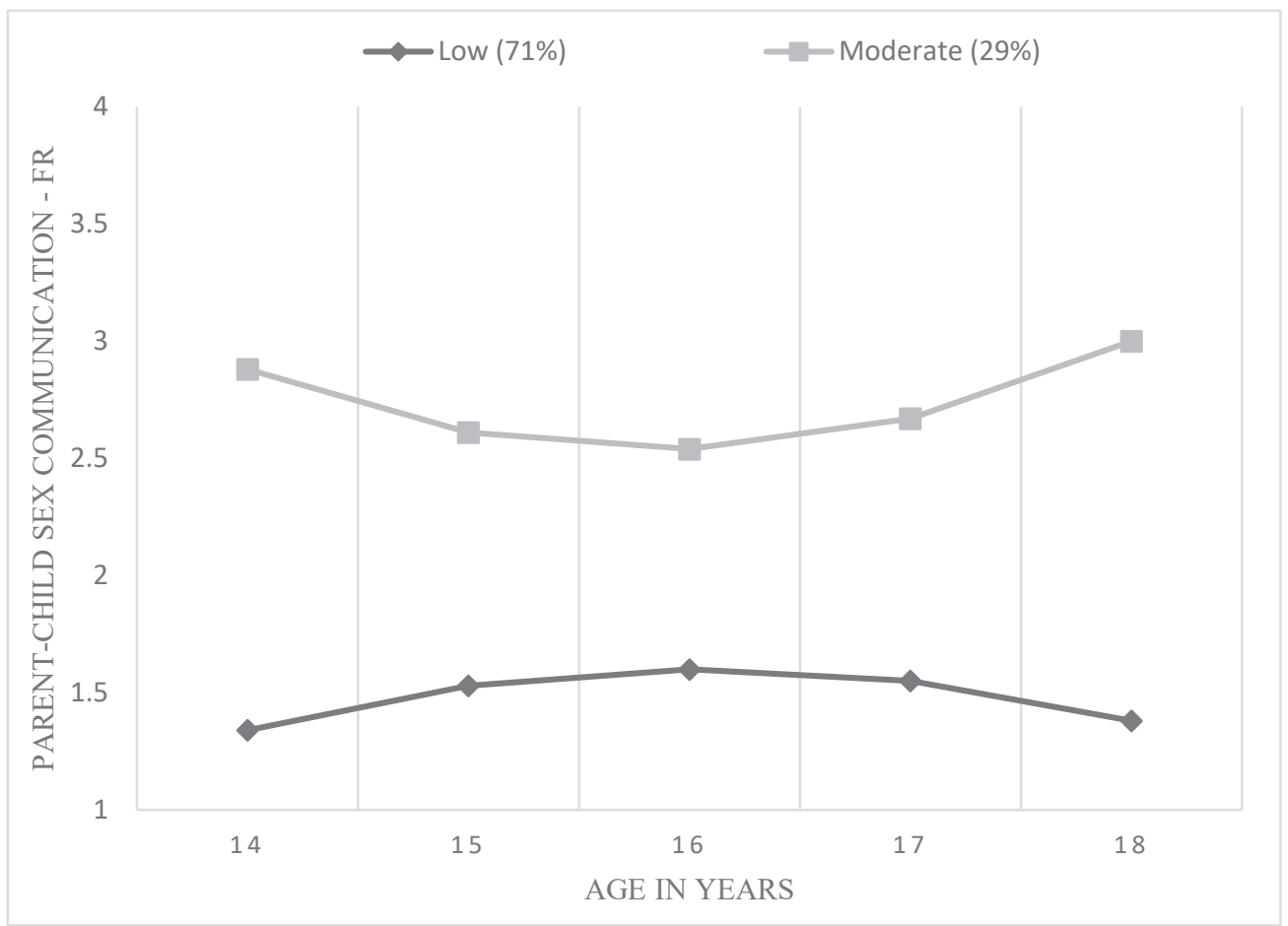

Figure 3. Growth mixture model of father-reported parent-child sex communication. 
Next, the du3step approach in Mplus was used to examine if means on outcome variables (at age 21 years) differed as a function of class membership (Table 2). The two outcome variables used were retrospective reports of age of first intercourse and reports of safe sex practices at age 21 years. It is of note that for child-, mother-, and father-reported models, classes with the lowest frequency of parent-child sex communication reported the latest age of first intercourse. In addition, for the motherreported model only, Moderate Stable Communicators had children who reported higher levels of safe sex practices than Low Communicators.

\section{Discussion}

Taken together, our findings suggested that there was meaningful variability in trajectories of parent-child sex communication across all three reporters (child, mother, and father), although trajectories varied somewhat between the child and parent reports. The findings also suggested that parent-child sex communication trajectories were most consistently distinguished by the adolescent's behavior (e.g., early sexual debut and externalizing problems) and family factors (i.e., single parent and ethnicity), although parental control seemed important for fathers' trajectory of sex communication. Finally, mothers who reported communicating more often across adolescence had teens who reported higher levels of safe sex practices.

\section{Heterogeneity in parent-child sex communication over time}

Developmentally one would expect that given normative increases in sexual development and romantic relationships from early to middle adolescence [3,24], parents would initiate parent-child sex communication at increasing levels from early adolescence throughout middle to late adolescence (when most teens become sexually active with a partner). Despite finding meaningful variability in parent-child sex communication, the majority of parents (70\% child report, $51 \%$ mother report, and $71 \%$ father report) in the present study displayed negligible levels of sex communication, which is consistent with research suggesting that conversations about sexuality are rare and most

Table 2

Test of mean differences across outcome variables as a function of class

\begin{tabular}{lll} 
& $\begin{array}{l}\text { Age at first } \\
\text { intercourse, } \\
\text { mean (SE) }\end{array}$ & $\begin{array}{l}\text { Safe sex, } \\
\text { mean }(\mathrm{SE})\end{array}$ \\
\hline Child report & $16.53(.41)^{\mathrm{ab}}$ & $1.19(.16)$ \\
$\quad$ Class 1 (8\%) Sweet 16 & $16.07(.33)^{\mathrm{a}}$ & $1.28(.24)$ \\
Class 2 (7\%) Peak at 16 & $16.98(.13)^{\mathrm{b}}$ & $1.01(.05)$ \\
Class 3 (69\%) Low Stable & $15.90(.29)^{\mathrm{a}}$ & $1.20(.11)$ \\
Class $4(16 \%)$ Moderate Stable & $17.05^{* * *}$ & 3.89 \\
Overall $\chi^{2}$ & $17.19(.15)$ & .96 \\
Mother report & $16.17(.16)$ & 1.24 \\
Class 1 (51\%) Low & $20.14^{* * *}$ & $8.82^{* *}$ \\
Class 2 (49\%) Moderate Stable & $17.22(.14)$ & $1.03(.06)$ \\
Overall $\chi^{2}$ & $16.45(.23)$ & $1.18(.10)$ \\
Father report & $7.50^{* *}$ & 1.63 \\
Class 1 (71\%) Low & & \\
Class 2 (29\%) Moderate & & \\
Overall $\chi^{2}$ & &
\end{tabular}

For Child-Reported Model, means with differing subscripts across rows are statistically different at $p<.05$.

${ }^{*} p<.05, * * p<.01, * * * p<.001$. often consist of a one-time "birds and the bees" talk during the early teenage years $[1,25]$, with little change over time [4]. That being said, the present study is the first to identify different trajectories of sex communication over time, suggesting that prevention/intervention programs designed as "one size fits all" might not be optimally effective. More specifically, the findings suggested that for child (16\%), mother (49\%), and father (29\%) reports, there was a portion of parents who seemingly talked with their children at least at the level of "sometimes" across ages 14-18 years, which is especially hopeful if mother reports are at all accurate. Child reports identified two additional small groups, one in which parents seemingly had some initial conversation about sexuality and then decreased communication until after age 16 years (Sweet 16\%, 8\%) and another in which parents discussed sexuality at increasing levels until age 16 years and then decreased to almost no communication by age 18 years (Peak at 16\%, 7\%). It is likely that these groups of parents would benefit from different approaches to education about parent-child sex communication than the "average" parent who engages in very low levels of sex communication.

In attempting to make sense of parents' reasons for stability or change in their levels of sex communication over time, we explored predictors associated with these trajectories. In aggregate, these findings suggested that parents engaged in more sex communication when risk was higher, including being non-white and from a single-parent family and adolescents who had an early sexual debut and higher levels of externalizing problems at age 14 years. This is consistent with past crosssectional research [9] but extends this research by suggesting that early risk shapes parental trajectories over time. Prevention and intervention programs may already encourage parents whose children are at high risk to engage in communication about sex and should continue to do so as the present study suggested that moderate levels of mother-child sex communication over time were effective at reducing sexual risk in children at age 21 years. However, the current findings suggest that lower risk adolescents are still getting very low levels of sex communication and may be less prepared at sexual debut. Furthermore, although safety outcomes are important to consider, both low- and high-risk teens need accurate information to foster healthy sexuality outcomes more broadly measured (including low sexual anxiety, high sexual esteem, knowledge of consent, etc), whether or not they are currently sexually active. Another important finding was that being a father who was a low communicator was predicted by high levels of maternal control (and low paternal control), which may be explained by fathers disengaging if they feel like the mother is gatekeeping [26]. This also has important implications for prevention and intervention efforts, suggesting that one way to get fathers more involved, which is sorely needed [1], is to help mothers to be less controlling.

Taken together, these findings contribute meaningfully to existing research that has explored the average level of parentchild sex communication and suggest that although the majority of parents do not vary in their low frequency of communication across adolescence, a sizable minority (depending on reporter) do display patterns that contain fluctuations in sex communication over time. That being said, it is also of note that initial levels and trajectories of parent-child sex communication varied significantly as a function of reporter, with the most marked differences between child and mother reports. Research has found that mean levels of parent and child reports of sex 
communication are often significantly different [4,11], with parents reporting much higher levels of communication than teenagers, and this study extends this to suggest that adolescents may also perceive patterns of communication over time to be quite different and potentially more nuanced than do parents. This is possibly because of teenagers ignoring or not attending to parental messages because of the embarrassment and discomfort that surround these conversations [1], but it could also be that parents are overestimating the frequency and quality of sex communication. Continued research is clearly needed, and educators and health care providers should be aware of the nuances of these disparities when communicating with parents and teenagers.

\section{Limitations and conclusions}

Despite coming from the strength of a longitudinal design and the use of multiple reporters, the present study was not without limitations and provides a variety of important avenues for future research. Namely, the measure of parent-child sex communication was only a frequency scale and focused solely on sexual risk. Given research that has highlighted the importance of the quality of parent-child sex communication [8], as well as the effectiveness of conversations regarding parental values and other aspects of sexual development [27,28], future research should focus on developmental change in frequency, quality, and the messages conveyed in parent-child sex communication to more holistically capture family dynamics in this regard. Using this nuanced approach to assessing parent-child sex communication, future research should work to establish what developmentally appropriate change in parent-child sex communication might look like and how that varies as a function of family, parent, and child characteristics. The present study also only focused on sexual risk outcomes, and while appropriate given the measure of parent-child sex communication, sexual health goes beyond merely avoiding sexual risk. Future research should also explore additional aspects of sexual health such as sexual esteem, relationship satisfaction, and sexual anxiety.

These limitations notwithstanding, a person-centered approach adds to our understanding of the frequency of the average parent's sex communication across development by highlighting that there is clearly more than one approach that parents take to discussing sexuality with their children. These findings suggest that researchers, educators, and health care providers should understand that a "one size fits all" approach to encouraging parent-child sex communication may be less than optimally effective. Furthermore, low-risk families (in addition to high-risk families) need to be encouraged to engage in sex communication with their children, and parents and teens may need to be educated differently, given discrepancies between parent- and child-reported understandings of the patterns of sex communication. Finally, fathers who may be hesitant to talk about sexuality with their children could benefit from mothers who are less controlling. Although professionals should consider sexual health to extend beyond safe sex, it is notable that low levels of parent-child sex communication were not effective at attenuating sexual risk, whereas moderate rates (as reported by mother) were effective at lowering sexual risk well into the adolescents' transition to adulthood.

\section{Supplementary Data}

Supplementary data related to this article can be found at https://doi.org/10.1016/j.jadohealth.2020.04.031.

\section{References}

[1] Flores D, Barroso J. 21st century parent-child sex communication in the United States: A process review. J Sex Res 2017;54:532-48.

[2] Murray A, Ellis MU, Castellanos T, et al. Sexual health discussions between African-American mothers and mothers of Latino descent and their children. Sex Educ 2014;14:597-608.

[3] de Graaf H, Vanwesenbeeck I, Meijer S, et al. Sexual trajectories during adolescence: Relation to demographic characteristics and sexual risk. Arch Sex Behav 2009;38:276-82.

[4] Padilla-Walker LM. Longitudinal change in parent-adolescent communication about sexuality. J Adolesc Health 2018;63:753-8.

[5] Baumrind D. Current patterns of parental authority. Dev Psychol 1971;4 $1-103$.

[6] Baumrind D. Differentiating between confrontive and coercive kinds of parental power-assertive disciplinary practices. Hum Dev 2012;55: 35-51.

[7] Harris AL, Sutherland MA, Hutchinson MK. Parental influences of sexual risk among urban African American adolescent males. J Nurs Scholarsh 2013;45:141-50.

[8] Rogers AA, Ha T, Stormshak EA, Dishion TJ. Quality of parent-adolescent conversations about sex and adolescent sexual behavior: An observational study. J Adolesc Health 2015;57:174-8.

[9] Capaldi DM, Forgatch MS, Crosby L. Affective expression in family problem-solving discussions with adolescent boys. J Adolesc Res 1994;9: $28-49$.

[10] Jaccard J, Dittus PJ, Gordon VV. Parent-adolescent congruency in reports of adolescent sexual behavior and in communications about sexual behavior. Child Dev 1998;69:247-61.

[11] Lefkowitz ES. Beyond the yes-no question: Measuring parentadolescent communication about sex. New Dir Child Adolesc Dev 2002:43-56.

[12] Mauras CP, Grolnick WS, Friendly RW. Time for "the talk" . . . now what? Autonomy support and structure in mother-daughter conversations about sex. J Early Adolesc 2013;33:458-81.

[13] Somers CL, Paulson SE. Students' perceptions of parent-adolescent closeness and communication about sexuality: Relations with sexual knowledge, attitudes, and behaviors. J Adolesc 2000;23:629-44.

[14] Rogers AA. Parent-adolescent sexual communication and adolescents' sexual behaviors: A conceptual model and systematic review. Adolesc Res Rev 2017:2:293-313.

[15] Hutchinson MK, Montgomery AJ. Parent communication and sexual risk among African Americans. West J Nurs Res 2007;29:691-707.

[16] O'Sullivan LF, Dolezal C, Brackis-Cott E, et al. Communication about HIV and risk behaviors among mothers living with HIV and their early adolescent children. J Early Adolesc 2005;25:148-67.

[17] Enders CK. Applied missing data analysis. New York, NY: Guilford Press; 1998.

[18] Turchik JA, Garske JP. Measurement of sexual risk taking among college students. Arch Sex Behav 2009;38:936-48.

[19] Robinson CC, Mandleco B, Olsen SF, Hart CH. The parenting styles and dimensions questionnaire (PSQD). In: Perlmutter BF, Touliatos J, Holden GW, eds. Handbook of family measurement techniques. 3rd ed. Thousand Oaks: Sage; 2001:319-21.

[20] Barber B. Parental psychological control: Revisiting a neglected construct. Child Dev 1996;67:3296-319.

[21] Barber BK, Stolz HE, Olsen JA, Maughn SL. Parental support, psychological control, and behavioral control: Assessing relevance across time, culture, and method. Monogr Soc Res Child Dev 2005;70:1-137.

[22] Tofighi D, Enders CK. Identifying the correct number of classes in growth mixture models. In: Hancock GR, Samuelsen KM, eds. Advances in latent variable mixture models. Charlotte, NC: Information Age Publishing; 2008:317-41.

[23] Celeux G, Soromenho G. An entropy criterion for assessing the number of clusters in a mixture model. J Classif 1996;13:195-212.

[24] Abma J, Martinez GM. National health statistics report: Sexual activity and contraceptive use among teenagers in the United States, $2011-2015$. (report No. 104). U.S. Department of Health and Human Services, Centers for Disease Control and Prevention and National Center for Health Statistics website. 2017. Available at: https://www.cdc.gov/nchs/data/nhsr/ nhsr104.pdf. Accessed December 1, 2019. 
[25] Collins C, Angera J, Latty C. College aged females' perceptions of their fathers as sexuality educators. J Ethnogr Qual Res 2008;2: $81-90$.

[26] Fagan J, Barnett M. The relationship between maternal gatekeeping, paternal competence, mothers' attitudes about the father role, and father involvement. J Fam Issues 2003;24:1020-43.
[27] Cheshire E, Kaestle CE, Miyazaki Y. The influence of parent and parent-adolescent relationship characteristics on sexual trajectories into adulthood. Arch Sex Behav 2019;48:893-910.

[28] Miller BC, Norton MC, Fan X, Christopherson CR. Pubertal development, parental communication, and sexual values in relation to adolescent sexual behaviors. J Early Adolesc 1998;18:27-52. 